\title{
MICROFLUIDIC LABEL-FREE IMMUNOCHIP FOR EARLY DIAGNOSTICS OF BREAST CANCER USING FUNCTIONALIZED POROUS GRAPHENE
}

\author{
Md. Azahar Ali, Seval Oren, Yueyi Jiao, Yifei Wang, Zhen Xu and Liang Dong ${ }^{*}$ \\ Iowa State University, Ames, USA
}

\begin{abstract}
We demonstrate a label-free microfluidic immunosensor for early diagnostics of breast cancer (epidermal growth factor receptor or ErbB2) with a detection limit at the level of $1.0 \mathrm{fM}$. This device utilizes a uniquely structured immunoelectrode made of porous graphene foam (GF) modified by titanium dioxide nanofibers $\left(\mathrm{nTiO}_{2}\right)$ at its surface. The antibody of ErbB2 acts as a detection probe immobilized on the GF-nTiO 2 electrode via EDCNHS chemistry. This immunosensor provides a high sensitivity of $43.7 \mathrm{k} \Omega / \mu \mathrm{M}$ in a wide detection range, good stability, and fast response $(3 \mathrm{~min})$ for detection of ErbB2 antigen.
\end{abstract}

\section{INTRODUCTION}

The ability to detect breast cancer biomarkers at an early stage is urgently required for clinical diagnostics and treatment for patient survival. Human epidermal growth factor receptor (EGFR, ErbB2, or HER2) gene encodes a $185-\mathrm{kDa}$ transmembrane glycoprotein and a receptor tyrosine kinase with intrinsic tyrosine kinase activity. Overexpression of ErbB2 $(\sim 33 \%)$ is linked to increasing breast cancer metastasis [1]. X-ray mammography, magnetic resonance imaging, computed tomography, and ultrasound imaging are commonly used methods of detecting cancerous tumors. Nevertheless, these tools rely heavily on imaging to determine the tumor status, providing insufficient information on the onset of cancer or monitoring of early stage cancerous cells.

Recently, microfluidics-based immunosensors, in conjunction with sensitive nanomaterials and nanostructures, have shown a great potential to revolutionize early detection of cancerous cells and therapy practices, offering cost-effectiveness, high sensitivity and selectivity, and portability. In particular, the two dimensional material, graphene, has been utilized to realize immunosensors capable of quantifying breast cancer biomarkers with a detection limit of $1.0 \mathrm{pM}[2]$.

Here we report a microfluidic electrochemical immunosensor with a uniquely structured bioelectrode made of porous graphene foam (GF) modified by titanium dioxide nanofibers $\left(\mathrm{nTiO}_{2}\right)$. AntiErbB2 antibody is conjugated at the surface of $\mathrm{GF}-\mathrm{nTiO}_{2}$ electrode to detect breast cancer biomarker ErbB2 via antigen-antibody interactions. The electrochemical impedance spectroscopy (EIS) is employed to monitor changes in interfacial properties of the bioelectrode (e.g., charge transfer resistance) induced by specific protein adsorption on the electrode surface.

\section{DEVICE FABRICATION}

Fig. 1a depicts the structure of the proposed microfluidic immunosensor. The device has a T-shaped, GF-nTiO 2 -based working electrode functionalized with anti-ErbB2 and attached to the upper side of a microfluidic channel through a pre-punched hole. A gold $(\mathrm{Au})$ counter electrode and an $\mathrm{Ag} / \mathrm{AgCl}$ reference electrode are formed on a glass slide (Fig. 1b). The immunoelectrode is placed above and in parallel with the counter electrode with a gap distance of about $500 \mu \mathrm{m}$. Biological functionalization of anti-ErbB2 on the surface of the $\mathrm{GF}^{-\mathrm{nTiO}}{ }_{2}$ composite is shown in Fig. 1c. The skeletons of porous GF is covered with electrospun $\mathrm{nTiO}_{2}$. $-\mathrm{COOH}$ groups are generated at the composite electrode via oxygen plasma treatment. This facilitates binding $-\mathrm{NH}_{2}$ groups of anti-ErbB2 with amide bond (CO-NH) through amidation reaction using EDC-NHS covalent chemistry. The non-specific sites of anti-ErbB2/GF-nTiO 2 are blocked by treating bovine serum albumin (BSA) molecules.

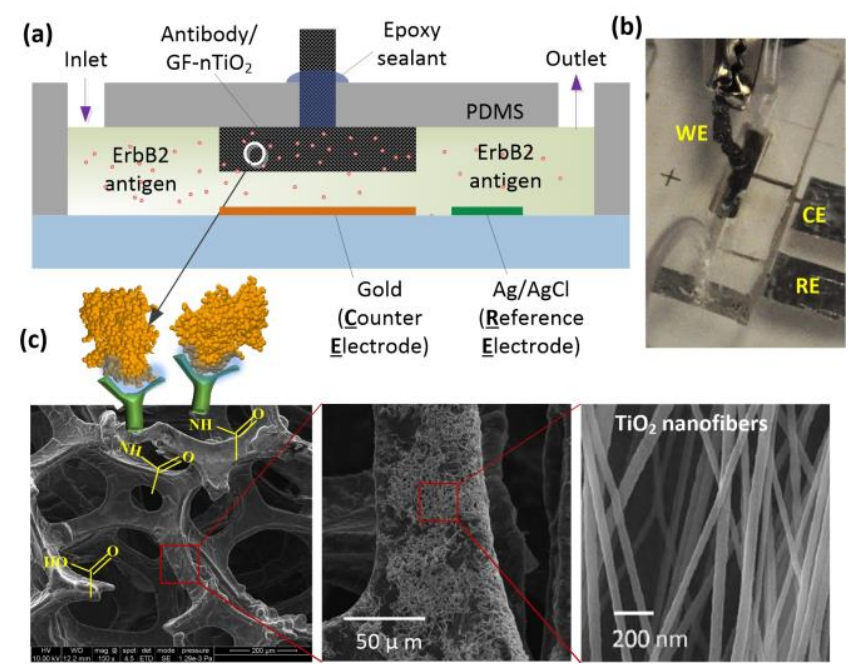

Figure 1: (a) Schematic (cross-sectional view) for a microfluidic electrochemical immunosensor for detection of breast cancer biomarker using a porous GF modified by $\mathrm{TiO}_{2}$ nanofibers. (b) Photo of the microfabricated immunosensor. (c) Pictorial representation of the proposed porous immunoelectrode, where anti-ErbB2 are attached to $\mathrm{nTiO}_{2}$ enabled GF. The SEM images shows the hierarchical structure of GF covered by $\mathrm{nTiO}_{2}$.

\section{RESULTS AND DISCUSSION}

Fig. 2 shows the Nyquist plots obtained by EIS for different electrodes, including GF, anti-ErbB2/GF, GF-nTiO2, and antiErbB2/GF-nTiO2 before and after being modified with BSA molecules. Measurements are conducted in presence of PBS buffer (pH: 7.4) containing $5 \mathrm{mM}\left[\mathrm{Fe}(\mathrm{CN})_{6}\right]^{3-/ 4-}$ as a redox probe. The charge transfer resistance $\left(R_{c t}\right)$ values of these electrodes are found based on the diameter of semicircle of the Nyquist plots. The GF electrode with antibody shows a higher $R_{c t}(45.3 \mathrm{k} \Omega)$ compared to that without antibody $(29.8 \mathrm{k} \Omega)$. The modification of GF electrode with $\mathrm{nTiO}_{2}$ increases $\mathrm{R}_{\mathrm{ct}}$ to $87.7 \mathrm{k} \Omega$ compared to the GF electrode. It should be noted that the larger surface area of the GF provides an easy access of $\mathrm{nTiO}_{2}$ suspension into its interior scaffolds, resulting in an enhanced loading capacity of anti-ErbB2 and thus a high readout signal. After immobilized with anti-ErbB2 and BSA, the immunoelectrode has an increased $\mathrm{R}_{\mathrm{ct}}$ of $152.6 \mathrm{k} \Omega$, due to the insulating nature of the BSA proteins that hinder redox conversion. Moreover, the anti-ErbB2 and BSA molecules immobilized on the GF-nTiO2 electrode lead to a higher surface coverage $(80.7 \%)$ compared to the bare GF-nTiO 2 electrode $(65.6 \%)$. This may help enhance sensitivity and detection limit of the sensor.

The EIS technique is utilized for detection of ErbB2 by varying its concentration from $1 \mathrm{fM}$ to $0.1 \mu \mathrm{M}$. Fig. 3 shows that the value of $R_{c t}$ increases with increasing concentration of ErbB2. 
Table 1: Comparison for sensing performances of our sensor with other electrochemical sensors reported in literature

\begin{tabular}{|l|l|l|l|l|l|}
\hline Electrodes & Techniques & Biomarkers & Sensitivity $\left(\boldsymbol{\mu M ^ { - 1 } )}\right.$ & Range $(\boldsymbol{\mu M})$ & Ref. \\
\hline $\mathrm{ZnO}$ nanofibers & Impedance & ErbB2 & $7.76 \mathrm{k} \Omega$ & $1 \times 10^{-9}-0.5$ & {$[1]$} \\
\hline $\begin{array}{l}\text { Graphene oxide-SiO } \\
\text { nanoparticles }\end{array}$ & Conductance & HER2 & NA & $1 \times 10^{-6}-1.0$ & {$[2]$} \\
\hline Carbon nanotubes & Cyclic voltammetry & Anti-breast cancer agent & $8-925 \mathrm{nA}$ & $40-160$ & {$[3]$} \\
\hline $\mathrm{ZnO}$ nanowires $^{\text {vifferential pulse }}$ & BRCA1 & $6.36 \mu \mathrm{A}$ & $10-100$ & {$[4]$} \\
\hline $\mathrm{GF}-\mathrm{nTiO}_{2}$ & Impedance & ErbB2 & $43.7 \mathrm{k} \Omega$ & $1 \times 10^{-9}-0.1$ & This work \\
\hline
\end{tabular}

Essentially, the interaction between specific binding sites of antiErbB2 and ErbB2 on the sensor surface results in the formation of immunocomplex that obstructs transfer of electrons generated from redox conversion. The sensor demonstrates a high sensitivity of $43.7 \mathrm{k} \Omega / \mu \mathrm{M}$ with a low detection limit of $1.0 \mathrm{fM}$ for the breast cancer biomarker. Also, the association constant of the immunoeletrode is as high as $4.46 \mathrm{kM}^{-1} \mathrm{~s}^{-1}$, indicating a high affinity towards ErbB2 antigen.

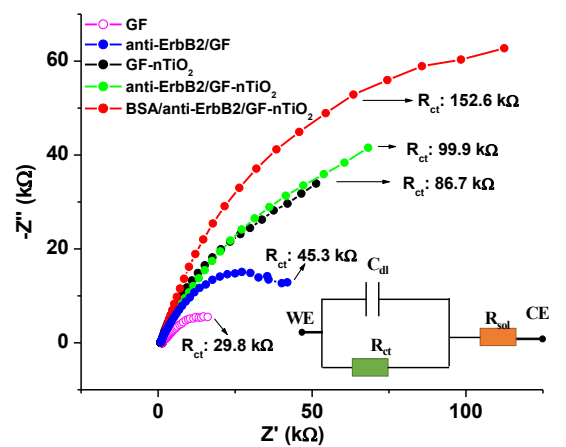

Figure 2: EIS spectra for five electrodes, including $G F$, antiErbB2/GF, GF-nTiO2, and anti-ErbB2/GF-nTiO2 before and after modified with BSA molecules. Inset shows an equivalent circuit model for the Nyquist plot to obtain the value of $R_{c t}$.
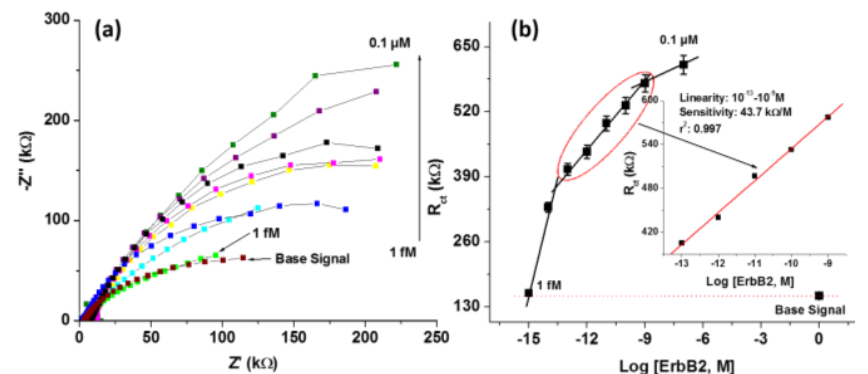

Figure 3: (a) EIS spectra of the sensor for detecting breast cancer biomarker (ErbB2) as the concentration of ErB2 is varied from 1.0 $f M$ to $0.1 \mu M$. (b) Calibration plot for charge transfer resistance $\left(R_{c t}\right)$ as a function of concentration of ErbB2.

Fig. 4a demonstrates the selectivity of the sensor in presence of human IgG, cholesterol, and BSA molecules as is evident by its low relative standard deviation (RSD: $\pm 2.0 \%$ ). Fig. $4 \mathrm{~b}$ demonstrates the stability of the sensor (RSD: $\pm 1.0 \%$ ) within 35 days. A minor deviation of $\pm 4.5 \%$ in impedance changes is observed for four identical sensors, suggesting reasonable reproducibility. The response time for this sensor is about $180 \mathrm{sec}$ (inset of Fig. 4b).

Table 1 compares the immunosensing performance of the sensor with its comparison with other electrochemical sensors reported in literature [1-4]. Due to different mechanisms and different biomarkers, point-to-point comparisons are difficult. However, with the same antibody ErbB2, our device shows a sensitivity $(43.7 \mathrm{k} \Omega / \mu \mathrm{M})$ about six times of magnitude higher than that using $\mathrm{ZnO}$ nanofibers-based immunoelectrode $(7.76 \mathrm{k} \Omega / \mu \mathrm{M})$.
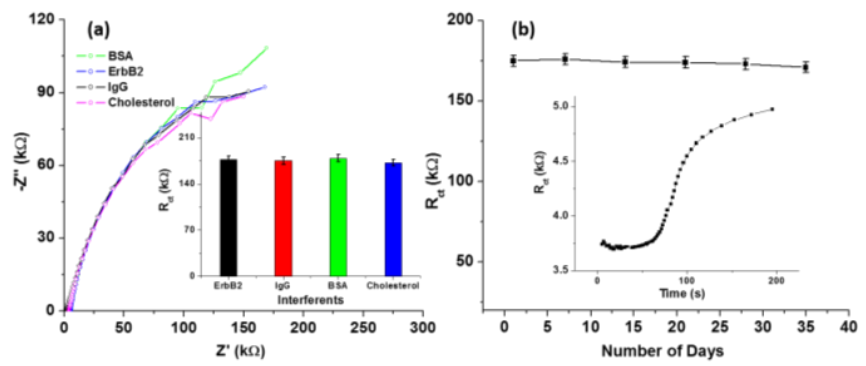

Figure 4: (a) EIS curves to investigate for interference studies of the fabricated immunosensor using different interferents (BSA, $\mathrm{IgG}$, and cholesterol) in presence of $100 \mathrm{nM}$, ErbB2, inset showing the $R_{c t} v$ interferents. (b) Response obtained for stability test of the sensor over 35 days. Inset shows the plot of dynamic change in impedance as time.

\section{ACKNOWLEDGEMENTS}

This work was supported in part by the National Science Foundation under grant ECCS-0954765 and the Iowa State University's Plant Sciences Institute Faculty Scholar Program.

\section{REFERENCES}

[1] M.A. Ali, K. Mondal, C. Singh, B.D. Malhotra, and A. Sharma, "Anti-epidermal growth factor receptor conjugated mesoporous zinc oxide nanofibers for breast cancer diagnostics", Nanoscale, 7, 7234 (2015).

[2] S. Myung, A. Solanki, C. Kim, J. Park, K.S. Kim and K.B. Lee, "Graphene-encapsulated nanoparticle-based biosensor for the selective detection of cancer biomarkers", Advanced Materials. 23, 2221 (2011).

[3] C. Baj-Rossi, G.D. Micheli, and S. Carrara, "Electrochemical detection of anti-breast cancer agents in human serum by cytochrome P450-coated carbon nanotubes", Sensors, 12, 552 (2012).

[4] N. A. Mansor, Z. M. Zain, H. H. Hamzah, M.S.A. Noorden, S.S. Jaapar, V. Beni, and Z.H. Ibupoto, "Detection of breast cancer 1 (BRCA1) gene using an electrochemical DNA Biosensor based on immobilized $\mathrm{ZnO}$ nanowires", Journal of Applied Biosensor, 3, 9-17 (2014).

\section{CONTACT}

*L. Dong, tel: +1-515-294-0388; 1dong@iastate.edu 